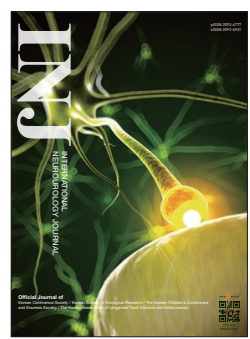

\title{
Research on Novel Intravesical Drug Delivery Devices
}

\author{
Hong Sang Moon (iD http://orcid.org/0000-0003-2101-1019 \\ Department of Urology, Hanyang University College of Medicine, Seoul, Korea \\ E-mail: moonuro@hanyang.ac.kr
}

Urologists usually treat overactive bladder (OAB) with oral medications. However, when there is no response to medication, alternative methods of treatment, including botulinum toxin A injection and sacral neuromodulation, are utilized. Nevertheless, botulinum toxin A requires repeated injections, causes adverse effects such as acute urinary retention, and reduces patient's compliance to treatment due to the discomfort of multiple rounds of injection-based therapy. Sacral neuromodulation can result in problems like lead migration, pain, and infection.

Whether given orally or via injection, therapeutic drugs may induce adverse effects in other organs aside from the target organs due to their systemic distribution. Long-term drug treatment may be difficult in some cases due to adverse effects; however, this problem could be overcome by drug delivery directly to the target organ in order to minimize systemic effects.

Treatment with antimuscarinic agents can induce problems among $\mathrm{OAB}$ patients by causing dry mouth and constipation. These adverse effects reduce patient's compliance, thereby decreasing treatment efficacy. In an attempt to overcome this problem, researchers have attempted direct instillation of oxybutynin or lidocaine into the bladder, with results showing improvement in lower urinary tract symptoms (LUTS) [1]. However, this procedure requires repeated drug instillation using a urethral catheter, and the effects are neither continuous nor lasting.

Based on the idea that devices that release drugs directly into the bladder may have potential to become a new treatment modality, several devices have been designed. One of the first devices created was the UROS bladder pump [2]. This device is a straight tube that is inserted into the bladder via cystoscopy and releases oxybutynin directly inside the bladder. Another device, the LiRIS, was designed by Lee and Cima [3]. The LiRIS device is a silicone tube, which is inserted into the bladder via cystoscopy and allows lidocaine to elute into the bladder. Research is being done aiming to create other effective tubular shaped treatment devices that facilitate drug elution into the bladder for extended periods.

Implanting a device into a patient's body may be a satisfactory therapeutic modality for $\mathrm{OAB}$, as it should theoretically have high efficacy while minimizing adverse effects [4]. Nonetheless, there are several issues associated with implantation of a device into the body. First, this requires an invasive procedure to insert the device. In some cases, general anesthesia or local anesthesia may be necessary, which increases anesthetic risks and patient discomfort. Second, device insertion into the bladder may create problems such as hemorrhage, infection, and increased expense, and third, the device may worsen LUTS by irritating the bladder. Even though symptomatic improvement has been shown after device insertion, the duration of efficacious treatment after insertion remains to be determined. In addition, this procedure needs periodic device replacements, which gives rise to challenges including patient discomfort, higher cost, and adverse effects. Moreover, if the device is placed inside the bladder permanently, device encrustation may occur.

Implantable devices in the bladder should fulfill several prerequisites. There should be minimal discomfort during and after implantation, and the device should not be expelled during urination. The design of the device should maximize therapeutic value by facilitating release of optimal levels of drug, mini- 


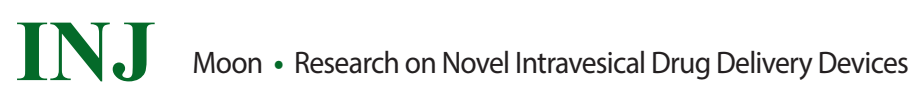

mizing adverse effects, reducing the need for repeated device insertion and removal, and preventing infection or device encrustation. Currently, a device that meets all of these requirements has not yet been invented.

At present, there is few effective and convenient treatments for intractable OAB. It is hoped that collaboration between urologists and biomedical engineers may create a treatment modality that maximizes effects while minimizing discomfort.

- Conflict of Interest: No potential conflict of interest relevant to this article is reported.

\section{REFERENCES}

1. Matsuoka PK, Haddad JM, Pacetta AM, Baracat EC. Intravesical treatment of painful bladder syndrome: a systematic review and meta-analysis. Int Urogynecol J 2012;23:1147-53.

2. Fraser MO, Lavelle JP, Sacks MS, Chancellor MB. The future of bladder control-intravesical drug delivery, a pinch of pepper, and gene therapy. Rev Urol 2002;4:1-11.

3. Lee H, Cima MJ. An intravesical device for the sustained delivery of lidocaine to the bladder. J Control Release 2011;149:133-9.

4. Lee SH, Choy YB. Implantable devices for sustained, intravesical drug delivery. Int Neurourol J 2016;20:101-6. 